\title{
GAIN STUDIES ON PHOTOCONDUCTORS MADE ON PARTLY COMPENSATED GaAs*
}

\author{
F. RIESZ \\ Research Institute for Technical Physics of the Hungarian Academy of Sciences \\ P. O. Box 76, 1325 Budapest, Hungary
}

The gain behavior of GaAs photoconductors realized on the partly compensated channel of a MESFET is studied. The gain versus light power dependence hints at the domination of the bimolecular recombination and the trap-mediated gain, and only a minor role of the surface photovoltaic effect. The possible correlation between dark current and gain mechanism is pointed out.

PACS numbers: $73.50 . \mathrm{Pz}, 85.60 . \mathrm{Gz}$

The photodetection, and in particular, gain mechanisms in compound semiconductor photoconductive detectors is a field of intense research [1]. Gain studies that have been published so far concentrated on photoconductors made of MESFET-like $n$-type epilayers. However, semi-insulating or other compensated materials are often used to ensure high-speed operation and low dark current [2]. In this work, we report on the gain behavior of GaAs photoconductors realized on partly compensated material.

The device is essentially a gateless MESFET, where the channel and a part of the buffer layer is removed by chemical etching [3]. The layer structure consists of a $3 \mu \mathrm{m}$ nominally undoped buffer layer and a $0.3 \mu \mathrm{m} n$-type layer doped to $4 \times 10^{17} \mathrm{~cm}^{-3}$, all grown by vapor-phase epitaxy (VPE). The channel width is $200 \mu \mathrm{m}$; the separation between the metal contacts is $5.5 \mu \mathrm{m}$, but the actual channel length is about $10 \mu \mathrm{m}$ due to the etch undercut at the contacts. The contacts are of alloyed $\mathrm{AuGe} / \mathrm{Ni} / \mathrm{Au}$. Etching was performed down to $0.7 \mu \mathrm{m}$ as checked by scanning electron microscopy. Prior to etching, the device $I-V$ (current-voltage) curves indicated that the contacts are indeed ohmic (injecting).

The optical gain was inferred from the measured dark and illuminated current-voltage curves. For illumination, a $785 \mathrm{~nm}$ laser diode was used whose intensity was measured with a calibrated Si photodiode. We estimated that $10 \%$ of the total incident power is absorbed in the device gap.

It is reasonable to write the dependence of gain $G$ on optical power $P_{\text {opt }}$ as

$$
G=G_{0}\left(P_{\mathrm{opt}} / m W\right)^{\beta} \text {, }
$$

*This work is supported in part by the (Hungarian) National Scientific Research Fund (OTKA) through grant F 4138. 
since, as we will discuss later, most theories predict such a power relationship. Any deviance from this rule can be taken into account by assuming a dependence of $\beta$ on $P_{\text {opt }}$.

Three mechanisms have been proposed to explain the light-power dependence of gain: (1) the photovoltaic (PV) effect at the surface [1]; (2) the bimolecular nature of the recombination process [4] and (3) a trap-mediated gain [4]. The PV effect is the modulation of the surface barrier by light: at higher barriers, the lifetime and consequently, gain, become higher because the barrier separates the photocarriers. The exponent $\beta$ equals the minus ideality factor of the surface diode (that is, $\beta$ is close to -1 ), but the gain saturates at lower optical powers. The PV effect dominates in highly doped (e.g. MESFET-like) layers, where large surface barriers exist. The bimolecular process is dominant when the generated carrier density exceeds the intrinsic carrier concentration; $\beta=-0.5$ in this case. If traps assist in the gain process, $\beta=0 \ldots-0.5$ depending on the trap density and their energy distribution [4].

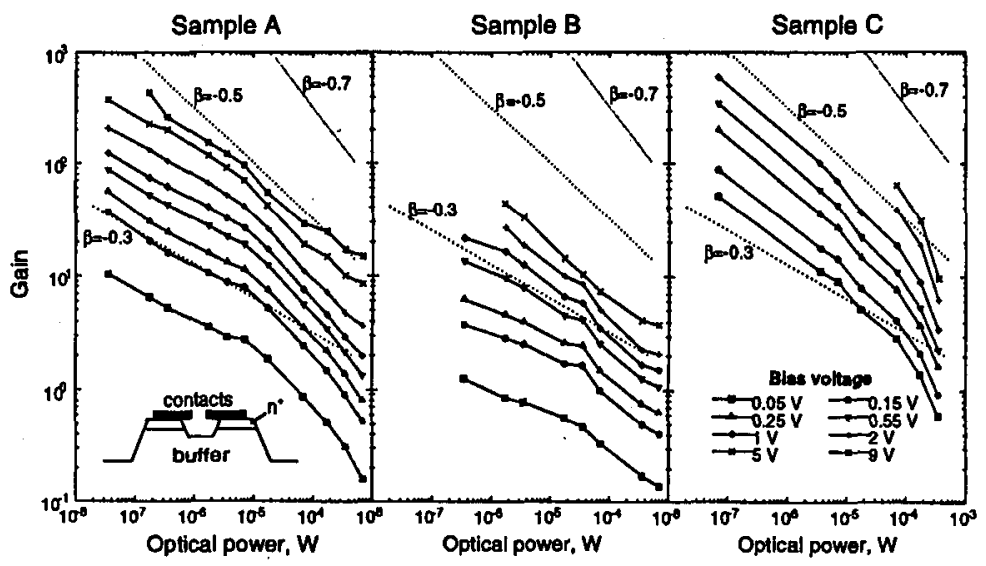

Fig. 1. Optical gain as a function of the total (incident) optical power for three representative devices. The straight lines for some $\beta$ values are also shown. Inset shows the schematic cross-section of the device.

We found that in a particular device, $\beta$ only slightly depends on bias but its absolute value increases with increasing optical power. Detailed data are shown here only for three representative samples (Fig. 1). The corresponding dark current curves are shown in Fig. 2. Although we cut all devices from the same wafer, etched to the same depth, our devices differ because of the inherent spatial inhomogeneity of our VPE process [5].

At a first glance, our data show $\beta$ values close to -0.5 , hinting at the dominance of the bimolecular recombination. However, in samples $A$ and $B, \beta$ approaches even -0.3 , indicating a trap-assisted gain. The traps also caused a long tail in the pulse response of our devices [3]. The correlation with dark current is worth noting: the traps that cause the $\beta \approx-0.3$ values, decrease also the dark current by trapping mobile carriers. The PV effect probably plays only a minor role 


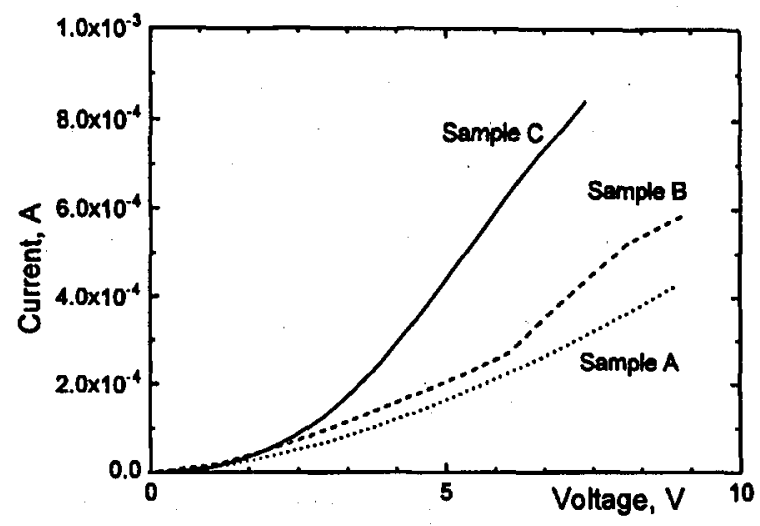

Fig. 2. The dark current of our sample devices.

in our devices. According to Darling [6], at higher optical powers, light-induced surface band bending can occur even in Cr-doped semi-insulating GaAs due to the charge redistribution in surface states. This may be responsible for the increasing $\beta$ (absolute) values at higher optical powers in our devices. However, heating effects that decrease the gain [7] cannot be ruled out.

The device parameters also support the possible presence of the three above-mentioned gain mechanisms. The buffer layer is nominally undoped, giving rise to bimolecular recombination. However, a background doping is usually present in the layer, as we concluded from electrochemical profiling measurements [8], causing possible PV effects. Finally, deep levels (traps) are usually present in such VPE-grown buffers [9], partly, but not solely, related to $\mathrm{Cr}$ diffusion from the substrate. The device-from-device variation is a consequence of the inherent inhomogeneity of the layer thickness as well as free carrier and trap concentration.

In conclusion, we studied the gain behavior of GaAs photoconductors made on partly compensated material. The gain versus light power dependence hints at the domination of the bimolecular recombination and the trap-mediated gain, and only a minor role of the surface photovoltaic effect. Drawing more quantitative conclusions would require more information on the trap distribution in our layer structures as well as more experimental work. A detailed report will be published later.

\section{Acknowledgments}

The author is grateful to $M$. Németh-Sallay and her co-workers for device fabrication, V.V. Tuyen for assistance in the measurements, K. Somogyi and P. Gottwald for illuminating discussions and B. Szentpáli for encouragement and discussions. 


\section{References}

[1] G.J. Papaioannou, J. Appl. Phys. 72, 5296 (1992).

[2] B.A. Bell, A.G. Perrey, R.A. Sadler, IEEE Trans. Instrum. Meas. 38, 92 (1989); C.H. Lee, Appl. Phys. Lett. 30, 84 (1977); R.A. Lawton, A. Scavannec, Electron. Lett. 11, 74 (1975).

[3] F. Riesz, B. Szentpáli, P. Gottwald, M. Németh-Sallay, Microwave Opt. Technol. Lett. 5, 112 (1992).

[4] A. Rose, $R C A$ Rev. 12, 362 (1951).

[5] K. Somogyi, Sz. Varga, I. Gyúró, A. Nemcsics, Crystal Properties Preparation 32-34, 459 (1991).

[6] R.B. Darling, Phys. Rev. B 43, 4071 (1991).

[7] J.P. Vilcot, J.L. Vaterkowski, D. Decoster, M. Constant, Electron. Lett. 20, 86 (1984).

[8] B. Szentpáli, F. Riesz, P. Gottwald, G. Reisinger, Opto Elektronik Magazin 6, 400 (1990).

[9] K. Somogyi, Crystal Properties Preparation 12, 95 (1991); ibid. 32-34, 453 (1991). 